\title{
Computational Hyperspectral Unmixing using the AFSSI-C
}

\author{
Phillip K. Poon ${ }^{\mathrm{a}}$, Esteban Vera ${ }^{\mathrm{b}}$, and Michael E. Gehm ${ }^{\mathrm{b}}$ \\ ${ }^{a}$ College of Optical Sciences, University of Arizona, 1630 E. University Blvd, Tucson, AZ USA \\ 85719 \\ ${ }^{\mathrm{b}}$ Department of Electrical and Computer Engineering, Duke University, Box 90291, Durham, \\ NC USA 27708
}

\begin{abstract}
We have previously introduced a high throughput multiplexing computational spectral imaging device. The device measures scalar projections of pseudo-arbitrary spectral filters at each spatial pixel. This paper discusses simulation and initial experimental progress in performing computational spectral unmixing by taking advantage of the natural sparsity commonly found in the fractional abundances. The simulation results show a lower unmixing error compared to traditional spectral imaging devices. Initial experimental results demonstrate the ability to directly perform spectral unmixing with less error than multiplexing alone.
\end{abstract}

Keywords: Compressive Sensing, Computational Imaging, Computational Spectroscopy, Spectral Unmixing

\section{INTRODUCTION}

The Airborne Visible/Infrared Imaging Spectrometer (AVIRIS) is an example of a traditional hyperspectral imager. ${ }^{1}$ AVIRIS has a spatial resolution of approximately $20 \mathrm{~m}$ at a cruising altitude of $20 \mathrm{~km}$ above sea level. At this spatial resolution, multiple material spectra are frequently reflecting or radiating into the field of view (FOV) of each spatial pixel $(m, n) .^{2,3}$ This results in a mixed spectrum $\mathbf{x}$ which is a combination of one or more consitutent spectra, $\mathbf{s}_{i}$, called endmembers.

In spectral classification, the goal is to identify the presence of a single endmember from a spectral library, $\mathbf{S}=\left[\begin{array}{llllll}\mathbf{s}_{1} & \mathbf{s}_{2} & \ldots & \mathbf{s}_{p} & \ldots & \mathbf{s}_{P}\end{array}\right]$. Often the presense of a mixed spectrum prevents the use of direct classification algorithms and we must therefore turn to spectral unmixing. Spectral unmixing is an analysis method which estimates which endmembers are present in the mixed spectrum $\mathbf{x}$ and their corresponding fractional abundances a.

In traditional spectral imaging, the unmixing step can only begin after the entire spectrum for a pixel has been acquired. For a spectrum with $L$ spectral channels, this requires $L$ measurement steps in the case of the tunable filter. ${ }^{4}$ A computational spectral imager is not nessecarily bound by this constraint.

We have previously designed, built, and demonstrated a computational spectral imager, called the Adaptive Feature Specific Spectral Imaging-Classifier (AFSSI-C)..$^{5-7}$ The AFSSI-C measures psuedo-arbitrary spectral filters $\mathbf{f}$ onto the spectrum $\mathbf{x}$ at each spatial pixel $(m, n)$ at each measurement step $j$. We have recently showed that when compared to traditional spectral imaging techniques such as the tunable filter, the AFSSI-C architecture provides a multiplex advantage in spectral unmixing. ${ }^{8}$

This paper discusses our latest results, whereby using techniques borrowed from the compressive sensing community, ${ }^{9-12}$ it is possible to estimate the fractional abundance with a lower root-mean-square error (RMSE) compared to the least squares estimator (LSE). Compressive sensing provides the ability to estimate the fractional abundance in less measurements than the number spectral channels $J<L$, providing an advantage over the tunable filter.

Further author information: (Send correspondence to Michael E. Gehm)

Phillip K. Poon: E-mail: ppoon@optics.arizona.edu

Esteban Vera: E-mail: esteban.vera@duke.edu

Michael E. Gehm: E-mail: michael.gehm@duke.edu

Computational Imaging, edited by Abhijit Mahalanobis, Kenneth S. Kubala, Amit Ashok,

Jonathan C. Petruccelli, Lei Tian, Proc. of SPIE Vol. 9870, 987003 · (c) 2016 SPIE

CCC code: $0277-786 X / 16 / \$ 18 \cdot$ doi: $10.1117 / 12.2223193$

Proc. of SPIE Vol. $9870987003-1$ 


\section{THE ADAPTIVE FEATURE SPECIFIC SPECTRAL IMAGING CLASSIFIER}

The AFSSI-C architecture is shown in Fig. 1. An unknown target, represented by the hyperspectral datacube is located at the front focal length of a collimating lens. The collimated light is transmitted through a diffraction grating and a second lens which images the sheared hyperspectral datacube onto the digital micromirror device (DMD). The DMD is a rectangular array of mirrors. Each DMD mirror can be programmed to reflect the light towards the second half of the AFSSI-C or towards the beam dump. Light that is passed is then collimated and transmitted through a second diffraction grating, which reverses the dispersion and unshears the filtered hyperspectral datacube. Finally the light is imaged onto a focal plane array (FPA) of a grayscale camera. The readout of the camera is equivalent to integrating over the filtered spectral channels.

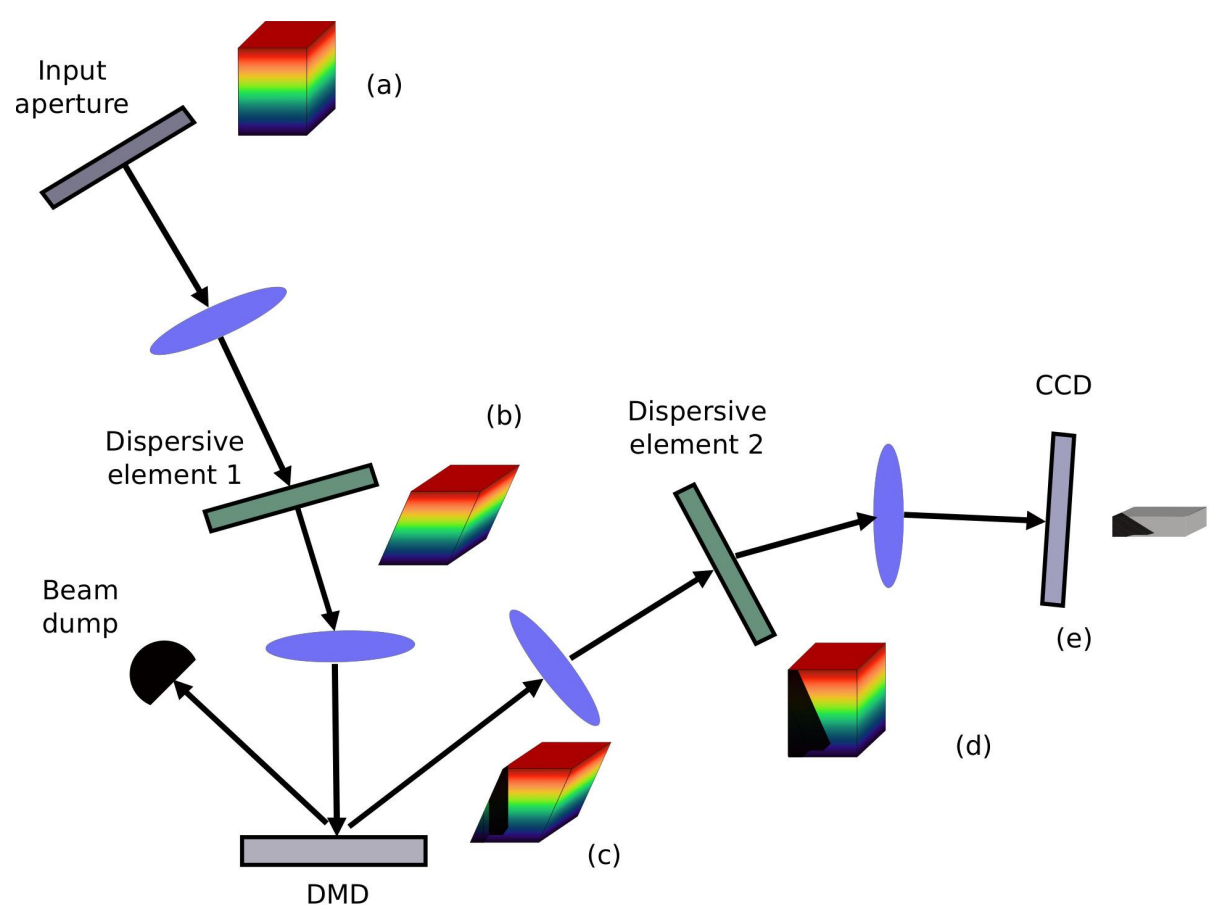

Figure 1: The optical architecture of the AFSSI-C: (a) The object or intermediate image is locate at the input aperture (b) The dispersive element then laterally shears and a second lens images this onto a DMD. (c) The act of reflecting from the hyperspectral datacube into the beam dump deletes vertical columns in the sheared datacube and the act of reflecting toward to the camera passes vertical columns. (d) The second dispersive element completely undoes the first and unshears the hyperspectral datacube. (e) The readout of the grayscale image from the camera is equivalent to a projection onto the spectral filter.

A single measurement step $j$ occurs when the spectrum $\mathbf{x}$ from a single pixel on the object $(m, n)$ is dispersed and imaged on $L$ DMD mirror groups (which are either passing or blocking light), then undispersed and readout on a single pixel on the FPA, which forms an inner product:

$$
g_{j}=\mathbf{f}_{j}^{\mathrm{T}} \mathbf{x}+\epsilon_{j}
$$

where $\mathbf{f}_{j}^{\mathrm{T}}$ is the transpose of the psuedo-arbitrary spectral filter created by imaging the dispersed spectrum $\mathbf{x}$ onto the DMD and $\epsilon_{j}$ is the additive noise. For multiple measurements $J$ we summarize Eq. 1 by matrix notation

$$
\mathbf{g}=\mathbf{F} \mathbf{x}+\epsilon
$$


where

$$
\mathbf{g}=\left[\begin{array}{c}
g_{1} \\
g_{2} \\
\vdots \\
g_{J}
\end{array}\right]
$$

and

$$
\mathbf{F}=\left[\begin{array}{c}
\mathbf{f}_{1}^{\mathrm{T}} \\
\mathbf{f}_{2}^{\mathrm{T}} \\
\vdots \\
\mathbf{f}_{J}^{\mathrm{T}}
\end{array}\right]
$$

\section{COMPUTATIONAL SPECTRAL UNMIXING}

In spectral unmixing, one simplification is that the mixed spectrum can be assumed to be a linear combination of endmembers

$$
\mathbf{x}=\sum_{p=1}^{P} a_{p} \mathbf{s}_{p}=\mathbf{S a} .
$$

We can justify this assumption in the case of a reflecting surface which exhibits a checkerboard mixture. ${ }^{13}$ In a checkerboard mixture, the fractional abundance of each endmember $a_{p}$ is linearly proportional to the fractional area of that material in the FOV of the FPA pixel. The incident light scatters only once with each material before being reflected into the FOV of the instrument. The forward model for the unmixing problem when applied to the AFSSI-C's architecture is written as

$$
\mathbf{g}=\mathbf{H a}+\epsilon,
$$

where $\mathbf{H}=\mathbf{F S}$.

With the forward model in place we can now discuss how to estimate the fractional abundance. In the tunable filter system $\mathbf{F}$ is the indentity matrix. Each spectral channel of $\mathbf{x}$ is measured independently. With each measurement having its own associated measurement noise and no multiplex advantage.

In natural scenes, the number of spectra that are actually present in the FOV of a FPA pixel is relatively sparse compared to the number of possible endmembers. This realization allows us to model the fractional abundance a with only a few non-zero elements. The Least Absolute Shrinkage and Selection Operator (LASSO) is suited for enforcing sparsity while estimating the fractional abundances via $\ell_{1}$ norm minimization:

$$
\hat{\mathbf{a}}=\underbrace{\arg \min }_{\mathbf{a}}\left\{\|\mathbf{x}-\mathbf{H a}\|_{2}^{2}+\|\mathbf{a}\|_{1}\right\}
$$

where $\hat{\mathbf{a}}$ is the estimated fractional abundance. ${ }^{11}$ Physically the fractional abundance must follow a sum-to-one constraint

$$
\sum_{p=1}^{P} a_{p}=1
$$

and a non-negativity constraint ${ }^{14}$

$$
a_{p} \geq 0 \quad \forall p
$$

We simulated a $64 \times 64 \times 40$ spectral datacube with a randomly generated fractional abundances for each pixel. Each fractional abundance has five endmembers but only two were present. Each fractional abundance satisfies the sum-to-one and non-negativity constraint. For each spatial pixel we generated a random spectral filter of dimension $L$ consisting of +1 's and -1's. We then average the results for a variety of signal-to-noise ratio (SNR) values 


$$
\mathrm{SNR}=\frac{\mathrm{E}\left[\operatorname{Var}_{L}\left[\mathbf{s}_{p}\right]\right]}{\sigma_{\text {noise }}^{2}}
$$

where $\operatorname{Var}_{L}\left[\mathbf{s}_{p}\right]$ is the variance of the endmembers over the spectral channels, $\mathrm{E}\left[\operatorname{Var}_{L}\left[\mathbf{s}_{p}\right]\right]$ is the average variance over all the endmembers, $\sigma_{\text {noise }}$ is the standard deviation of the additive Gaussian noise. The simulation shows that using the LASSO operator to promote sparse fractional abundances results in a lower average RMSE of approximately half a decibel, see Fig 2. RMSE is defined as

$$
\operatorname{RMSE}=\left[\frac{1}{P} \sum_{p=1}^{P}\left(\hat{a}_{p}-a_{p}\right)^{2}\right]^{\frac{1}{2}},
$$

where $\hat{a}_{p}$ is the $p^{t h}$ estimated fractional abundance and $a_{p}$ is the $p^{t h}$ inferred true fractional abundance.

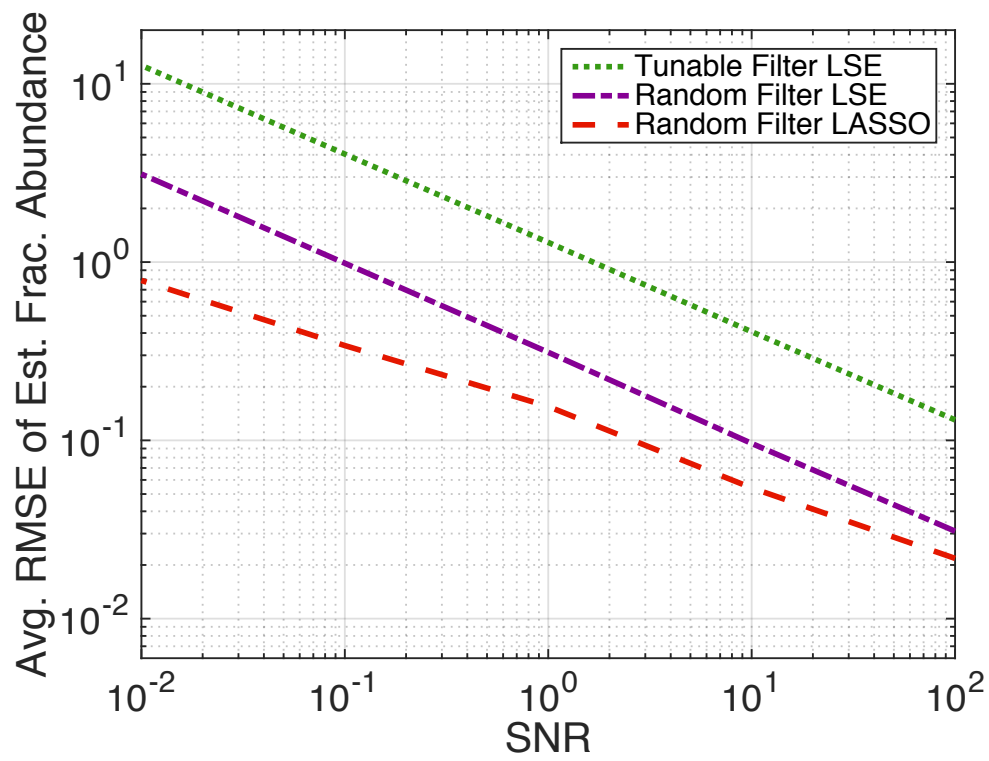

Figure 2: A comparision of the RMSE vs SNR after 40 measurements of the estimated fractional abundance for the tunable filter and random spectral filters with the LSE and LASSO estimators.

\section{INITIAL EXPERIMENTAL RESULTS}

In our experimental setup we create an artificially mixed spectrum by combining the light from a Dell P2311H LED Monitor and a 4D Systems uOLED-128G2 OLED display using a Thorlabs BP245B1 2 inch diameter pellicle beamsplitter, see Fig. 3a and Fig. 3b. An Edmunds $120 \mathrm{~mm}$ effective focal length (EFL) (Stock Number 32-921) archomatic doublet is used to move the OLED image location and image size so that it is approximately equal to the image location and size from the LED monitor. The rest of the experimental setup is part of the AFSSI-C instrument which is described in detail by 7 .

The LED monitor native resolution is $1920 \times 1080$. However only the center $512 \times 512$ physical pixels are used as part of the region of interest (ROI). This is further binned to $32 \times 32$ system pixels in the center of the monitor. The OLED display has a native resolution of $128 \times 128$. To make the alignment of the image from each display easier we only used one out of every four pixels. In other words in a $2 \times 2$ physical pixel square, only 1 out of 4 pixels is used. To match the resolution and image size from the LED monitor, we used the center $32 \times 32$ system pixels on the OLED display. The spectral library consists of 6 endmembers which are the red, green, and blue spectra from both displays, shown in Fig. 4. 
[a]

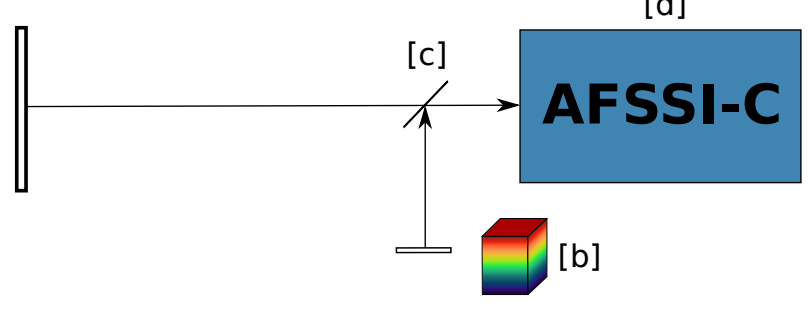

(a) [a] Light from the LED Monitor is combined with light from the OLED Display [b] using a beam splitter [c] to create an artificially mixed spectrum at the input aperture of the AFSSIC [d].

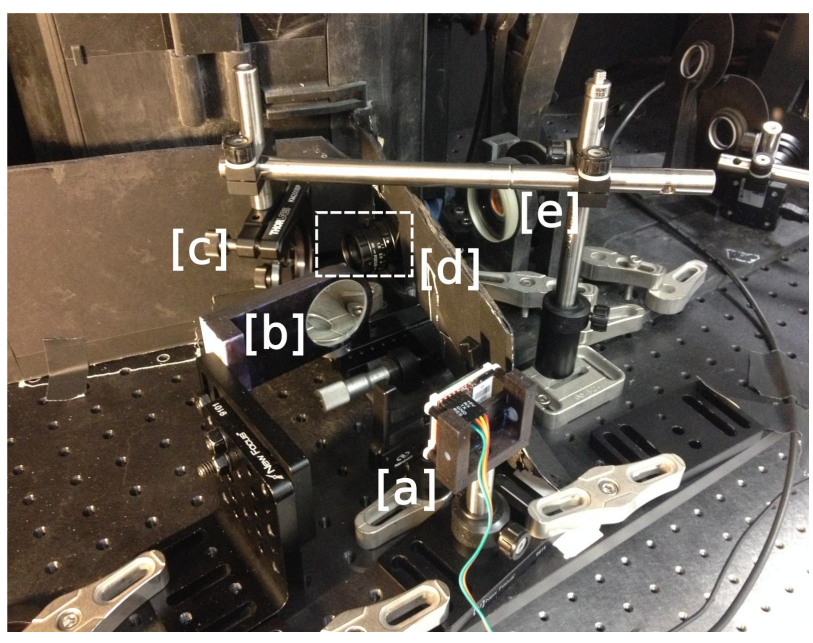

(b) [a] OLED display [b] $120 \mathrm{~mm}$ EFL achromatic doublet [c] pellicle beam splitter [d] $12 \mathrm{~mm}$ EFL machine vision lens [e] AFSSI-C Objective. The intermediate image plane of the combined (or mixed spectral) image is between the machine vision lens and the objective.

Figure 3: The experimental setup for creating a mixed spectrum.

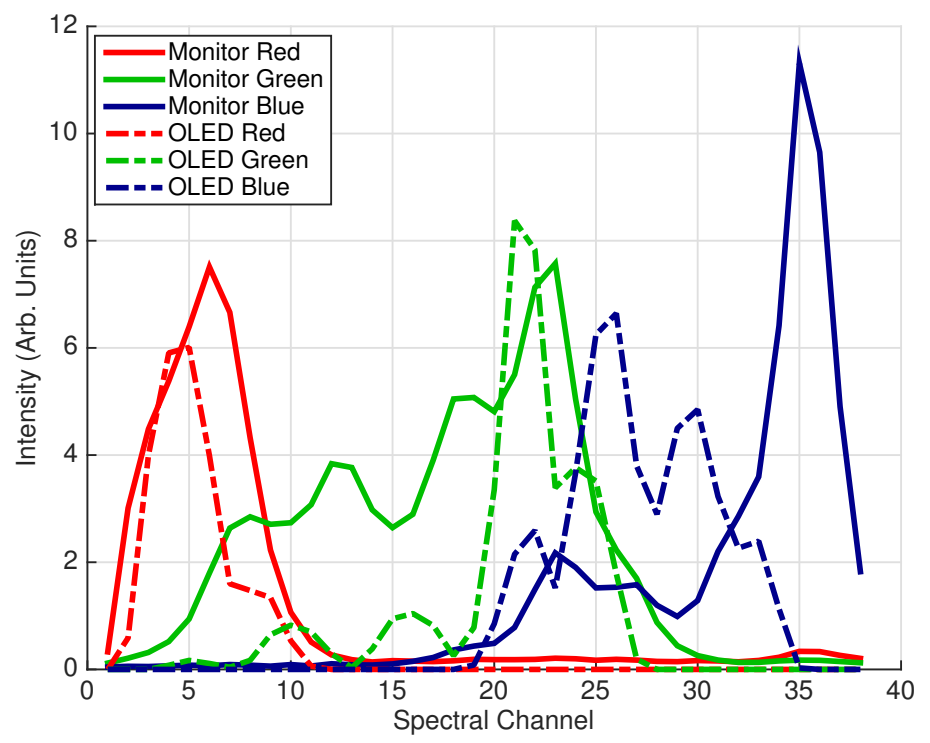

Figure 4: The spectral library used for the spectral unmixing experiment consists of the red, green, and blue from the LED monitor and an OLED display.

In our initial experiment we only turned on the green of the OLED display and the green of the LED monitor, thus the sparsity of the fractional abundance is 2 out of 6 . The intensity of the green on the LED monitor was set to ramp from 0.25 to 0.75 (in arbitrary units of intensity) from the left to right spatial columns and the intensity of the green on the OLED display was then set to ramp from 0.75 to 0.25 .

At each measurement step $j$ a random binary DMD code was generated which will be used as the spectral filters. Since the spectral filters consist of +1 's and -1 's and optical intensity cannot be negative, the spectral 
filters are seperated into positive and negative DMD codes.

$$
\begin{aligned}
& m^{+}=\mathbf{f}^{+\mathrm{T}} \mathbf{x}+\epsilon^{+} \\
& m^{-}=\mathbf{f}^{-\mathrm{T}} \mathbf{x}+\epsilon^{-}
\end{aligned}
$$

where $\mathbf{f}^{+\mathrm{T}}$ and $\mathbf{f}^{-\mathrm{T}}$ are the transpose of the positive and negative part of the spectral filter $\mathbf{f}$. Once the positive and negative measurements are stored digitally, we can compute the full measurement

$$
m=m^{+}-m^{-}
$$

The estimated weights were then normalized so they sum to one-yielding an estimated fractional abundance.

$$
\hat{\mathbf{a}}_{\text {norm }}=\frac{\hat{\mathbf{a}}}{\sum_{p=1}^{P} \hat{a}} .
$$

We plotted the normalized estimated fractional abundance for various locations in the image in Fig. 5. At pixel $(16,16)$, shown in Fig. 5a, the ratio of monitor green to OLED green approximates the inferred ground truth fractional abundance. The ratio of the two is approximately 1:1, which was the input. At another location (pixel $(20,29)$ shown in Fig. 5b) the normalized fractional abundance also approximates the inferred ground truth. In this case, as the number of measurements increase, the accuracy improves as intuition would suggest.

To get an idea of the overall error, we plotted the RMSE of the normalized estimated fractional abundance averaged over all $32 \times 32$ locations, see Fig. 6 . This plot was used to tune the $\lambda$ parameter of the MATLAB lasso function. Using the exact same spectral filters $\mathbf{F}$ and measurements $\mathbf{m}$, we can compare the performance of the LASSO with the Non-Negative Least Squares estimator (NNLS). The NNLS enforces the the non-negative solutions, however as we can see, promotting sparsity provides an additional advantage when we have passed a certain number of measurements.

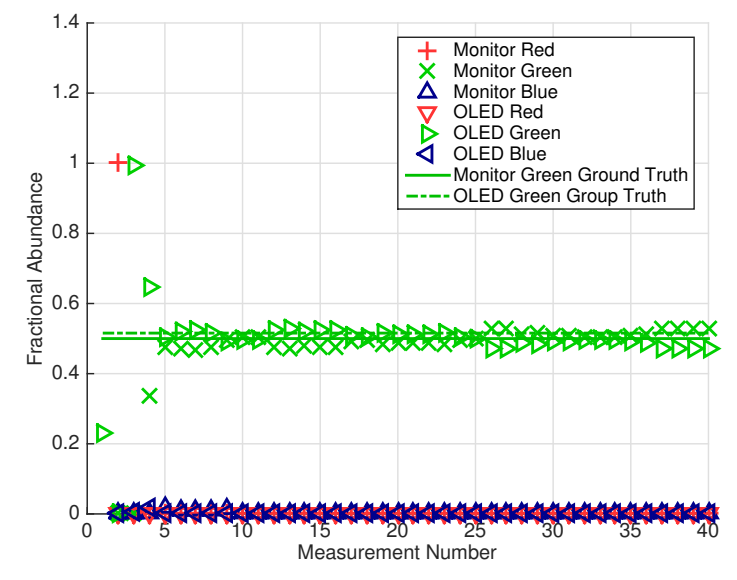

(a) Pixel $(16,16)$

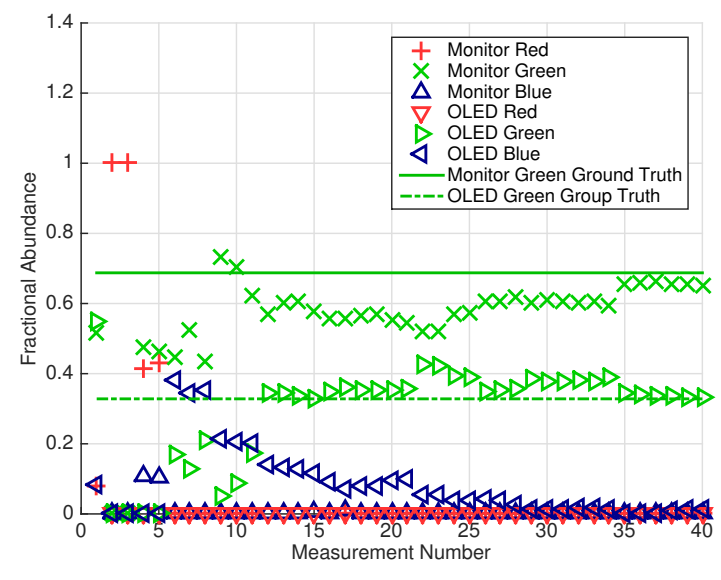

(b) Pixel $(20,29)$

Figure 5: The normalized estimated fractional abundances for various locations in the ROI using the LASSO operator.

\section{CONCLUSION}

Our simulation and preliminary experimental results show that by taking advantage of the natural sparsity commonly found in the fractional abundaces in mixed spectra we can achieve lower estimation error than multiplexing alone. Using the LASSO, the simulation results showed nearly half a decibel improvement compared to multiplexing with the LSE and approximately a full decibel improvement over traditional spectral imaging using the LSE. The experimental results show that LASSO can preform better than the NNLS given the same random binary spectral filters and measurement data. We believe that given further calibration and quanitifying the noise we can demonstrate experimental results that match the simulation results. Further work is being done to extend this to adaptive spectral unmixing as well demonstrating this on outdoor scenes. 


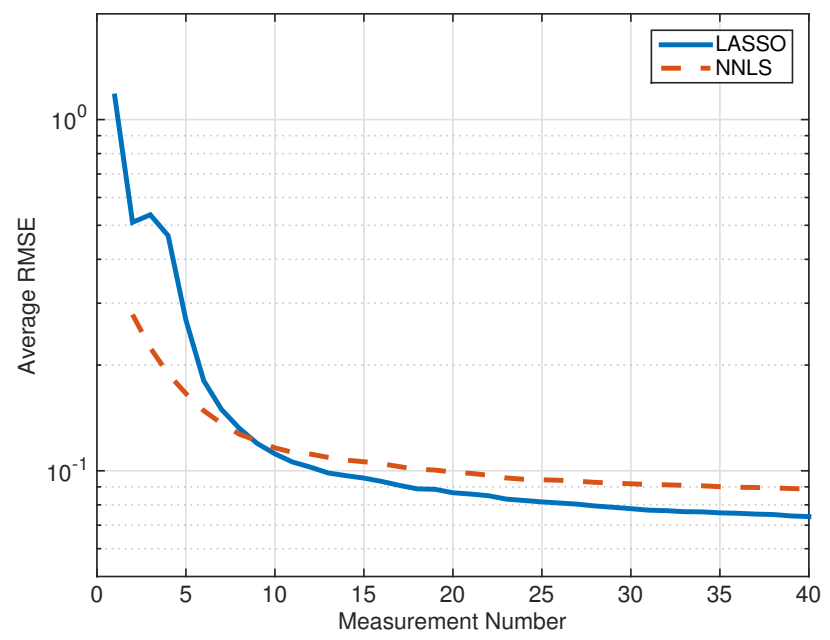

Figure 6: The LASSO operator has a lower average RMSE vs measurement number when compared to the NNLS estimator after less than 10 measurements. Each estimator used the same set of experimental measurement data g and spectral filters $\mathbf{F}$. The RMSE is averaged over all $32 \times 32$ pixels.

\section{Acknowledgments}

The authors would like to acknowledge Dr. Matthew Dunlop, Dr. Dathon Golish, and Dr. Peter Jansen who were part of the team that designed and developed the AFSSI-C.

We thank the DARPA KeCOM Project for prior financial support.

\section{REFERENCES}

[1] Vane, G., Green, R. O., Chrien, T. G., Enmark, H. T., Hansen, E. G., and Porter, W. M., "The airborne visible/infrared imaging spectrometer (AVIRIS)," Remote Sensing of Environment 44(2-3), 127-143 (1993).

[2] Boardman, J. W., "Automating spectral unmixing of AVIRIS data using convex geometry concepts," (1993).

[3] Goenaga, M. A., Torres-Madronero, M. C., Velez-Reyes, M., Van Bloem, S. J., and Chinea, J. D., "Unmixing analysis of a time series of hyperion images over the guánica dry forest in puerto rico," Selected Topics in Applied Earth Observations and Remote Sensing, IEEE Journal of 6(2), 329-338 (2013).

[4] Gat, N., "Imaging spectroscopy using tunable filters: a review," in [AeroSense 2000], 50-64, International Society for Optics and Photonics (2000).

[5] Dunlop, M., Jansen, P., Golish, D. R., and Gehm, M. E., "AFSSI-C: The Adaptive Feature-Specific Spectral Imaging Classifier," Imaging and Applied Optics Technical Papers, CM4B.4, Optical Society of America (2012).

[6] Dunlop, M., Poon, P. K., Golish, D. R., Vera, E., and Gehm, M., "Calibration challenges and initial experimental demonstration of an adaptive, feature-specific spectral imaging classifier," in [Imaging and Applied Optics], Imaging and Applied Optics, CW2C.3, Optical Society of America (2013).

[7] Dunlop, M., Poon, P., Vera, E., and Gehm, M. E., "Experimental validation of the adaptive feature-specific spectral imaging classifier," in [Frontiers in Optics 2014], Frontiers in Optics 2014, FTh2B.5, Optical Society of America (2014).

[8] Poon, P. K., Vera, E., and Gehm, M. E., "Hyperspectral unmixing using the AFSSI-C," in [Imaging and Applied Optics 2015], Imaging and Applied Optics 2015, IT2A.6, Optical Society of America (2015).

[9] Candes, E. J. and Tao, T., "Near-optimal signal recovery from random projections: Universal encoding strategies," Information Theory, IEEE Transactions on 52(12), 5406-5425 (2006).

[10] Donoho, D. L., "Compressed sensing," Information Theory, IEEE Transactions on 52(4), 1289-1306 (2006). 
[11] Tibshirani, R., "Regression shrinkage and selection via the lasso," Journal of the Royal Statistical Society, Series B 58, 267-288 (1994).

[12] Iordache, M.-D., Bioucas-Dias, J., and Plaza, A., "Unmixing sparse hyperspectral mixtures," in [Geoscience and Remote Sensing Symposium, 2009 IEEE International, IGARSS 2009], 4, IV-85, IEEE (2009).

[13] Keshava, N., "A survey of spectral unmixing algorithms," Lincoln Laboratory Journal 14(1), 55-78 (2003).

[14] Keshava, N. and Mustard, J. F., "Spectral unmixing," Signal Processing Magazine, IEEE 19(1), 44-57 (2002). 